\title{
A Determinant Analysis to Detect the Singularity of the Extended Theo Jansen Mechanism in the Phase-Rotation-Amplitude Parameter Space
} Kazuma Komoda ${ }^{1^{*}}$ and Hiroaki Wagatsuma ${ }^{1,2}$

${ }^{1}$ Department of Brain Science and Engineering, Graduate School of Life Science and Systems Engineering, Kyushu Institute of Technology, 2-4 Hibikino, Wakamatsu-Ku, Kitakyushu, Japan

${ }^{2}$ Laboratory for Dynamics of Emergent Intelligence, RIKEN Brain Science Institute, Hirosawa, Wako-shi, Saitama, Japan

\begin{abstract}
Legged robots have a potential of being a walking machine on irregular ground. Eleven-bar linkages, Theo Jansen mechanism reproduces a smooth locomotion pattern as gait. Parallel motions have widely used in the heavy machinery and recently highlighted in a model of biological motions. The close-loop linkage simply provides a designed end-effector trajectory, whereas the trajectory is considered to be less modifiable due to the singularity problem. In the present study, the singularity on the modified Theo Jansen mechanism was addressed by introducing the parametric orbit as a new freedom point in the joint center, and analyzed its kinematics and dynamics by using multibody dynamics (MBD). The extendability of the mechanism in the viewpoint of flexibility in the gait trajectory was clearly demonstrated in the numerical simulation, providing new functional gait trajectories controlled by two control parameters that change the shape of the parametric oval in the joint center. In systematic determinant analyses of how broken trajectories were generated depending on four parameters, i.e., horizontal and vertical amplitudes and rotation angle of the joint center movement and its phase difference with the driving link, morpho-logical changes of generated trajectories in the phase-rotation-amplitude parameter space were revealed. Thus the extension capability of Theo Jansen mechanism was validated not only in smooth walking but also in jumping, climbing and running-like motions. In considering the ways of control, the present results indicated that there exists a inverse relationship between the rotation angle and the phase difference to significantly reduce the occurrence of the singularity and breakage failures of the mechanism, which is consistent with biological evidences of coupling oscillators that enables the nervous system to control the complex musculoskeletal system by using a few of simple parameters frequently represented by the phase and rotation in a torus state space.
\end{abstract}

Keywords: Closed-loop linkage; Theo Jansen mechanism; Multibody dynamics; Determinant analysis; Gait trajectory

\section{Introduction}

Flexible and adaptive movements generated from biological systems are of interest not only to academics in the area of biomechanics but also to engineers to improve traditional mechanical schemes. Closed loop linkages are widely known because of the parallel motions used in the Watt steam engine to transform the motion from parallel, PeaucellierLipkin and Hart's inversor linkages to reproduce a completely straight line form cyclic motions by using different combinations of parallelograms. Bio-inspired mechatronics to mimic biological mechanisms in motions and behaviors have been investigated [1], and the legged motions are frequently modeled by the serial mechanism connected by joints with dampers and springs such as the passive walker [2], known as openloop linkages. The open-loop linkage is convenient for the generation of arbitrary trajectory of the end-effector with a supervision of every joint rotation angle governed by the central controller.

The bipedal locomotion is still a hot topic $[3,4]$; however the central controller paradigm either provides a heavy load of computation to calculate kinematics and dynamics of the system or requires a learning property of motion patterns. From the engineering viewpoint, it implies that the maxi-mum payload relies on the torque performance of individual actuators embedded in joints of the open-loop linkage. An alternative solution is closed-loop linkages, which have been widely used such as mechanical transmissions of explosion power in engines to wheel crankshaft in car and the walking ability in uneven ground $[5,6]$. Interestingly, Theo Jansen proposed a kinematics for providing of legged robots in the form of the closed-loop linkage with eleven linkbars, called Theo Jansen mechanism and demonstrated that it worked for heavy payload conditions in a various type of similar mechanisms
[7]. For analyses of the reproduced animal locomotion with smooth transition between swing and stand phases in a locomotion cycle, Giesbrecht [8] investigated an optimized orbit of legs from the viewpoint of a minimum energy input and a maximum stride length. Kim [9] demonstrated a real remote-controllable robot based on the mechanism to test the walking performance on shore and marsh place.

Theo Jansen aspired the ultimate gait trajectory and selected the best combination of linkage-bar lengths, which succeeded in providing a fixed locomotion pattern, whereas the mechanism stays in a position far from the alternative solution of legged systems based on the openloop linkage in the viewpoint of flexibility. In principle, closed-loop linkage has capabilities to change its motion even without changes of link-bar lengths due to additive motions of nodes. As efforts of extension of the original mechanism, Sitharam [10] investigated relationship between linkages by representing a connected component of the Euclidean realization space as a curve in a carefully chosen

*Corresponding author: Kazuma Komoda, Department of Brain Science and Engineering, Graduate School of Life Science and Systems Engineering, Kyushu Institute of Technology, 2-4 Hibikino, Wakamatsu-Ku, Kitakyushu 808-0196, Japan, Tel: 81936956159; E-mail: komoda-kazuma@edu.brain.kyutech.ac.jp

Received November 19, 2015; Accepted December 14, 2015; Published December 21, 2015

Citation: Komoda K, Wagatsuma H (2015) A Determinant Analysis to Detect the Singularity of the Extended Theo Jansen Mechanism in the Phase-RotationAmplitude Parameter Space. J Comput Sci Syst Biol 9:1 010-022. doi:10.4172 jcsb.1000215

Copyright: (c) 2015 Komoda K, et al. This is an open-access article distributed under the terms of the Creative Commons Attribution License, which permits unrestricted use, distribution, and reproduction in any medium, provided the original author and source are credited. 
non-edge distance parameter space and Nansai [11] proposed a wide variety of gait cycle by adding actuator to part of linkages. These studies demonstrated extension capabilities of the Theo Jansen mechanism by heuristic methods, but it lacks the comprehension and structural method of how it is possible.

Deadlocks and the breakage of the system kinematics in an inappropriate design is inevitable in the closed-loop linkage, while multibody dynamics (MBD), which is a technique for describing mechanism mathematically, clears a path to investigate the issue in a vistaed way. In the present study, an additional movable node was selected from fixed joints of the original Theo Jansen mechanism according to the inspiration of the toe-knee-inguinal relation of the human leg, which was preliminarily reported in our previous publication [12] and then we hypothesized that functional gait trajectories involving climbing and jumping were extensively associated with motion patterns of the movable node assumed as the knee position. The issue to be addressed in this paper is to clarify of the limitation of extendibility of the Theo Jansen mechanism to provide new gait patterns and the elucidation of characteristics on constraints of the parametric space of the additional node movement given by the parametric oval orbit.

We introduced determinant analyses of the system kinematics and investigated a safety assurance to avoid breaking in the parametric space. Thus, the present paper is devoted to validate the hypothesis on the extendability of the Theo Jansen mechanism through the generation of new and unbroken trajectories of the closed-loop linkage by using the parametric oval obit in the additional node with following four parameters. The amplitudes of the moving range in horizontal and vertical axes, the rotation angle of the oval orbit and the start position of the oval movement with respect to the synchronized movement of the input cycle rotation angle, i.e., the phase difference between the oval of the midpoint and the unit cycle of the input.

This paper is divided into the following sections: Section of "Hypothesis" addresses our hypothesis of how the original mechanism can be extended to multifunctional legged robots with a certain risk on the deadlock. Section of "Mathematical Formulations" describes the common framework of the model definition and preliminaries for multibody dynamics. Section of "Extension Model of the Theo Jansen Mechanism" describes the modeling formulation of a legged robot with closed-loop linkage known as the Theo Jansen mechanism. In Section of "Results of Numerical Simulations," characteristic analyses of the extended Theo Jansen mechanism are discussed with temporal evolutions of the placement, posture, velocity, acceleration, and determinant in three different conditions. In Section of "Functional Trajectory Generation Focusing on Amplitude Change," amplitude properties investigate for understanding behavior regarding adding orbits and leg's trajectories. Phase differences distribution is described in one circle case and two ellipse cases by changing amplitudes and rotation angle in Section of "Functional Orbit Generation Focusing on the Phase Difference." In Section of "Functional Orbit Generation Focusing on the Phase Difference," distribution of nonbroken trajectories has been classified in the extended Theo Jansen mechanism for getting non-broken trajectories. Effective conditions are explained regarding amplitude, phase differences, and rotation angle in the extended motion in discussion to realize functional motion. Coordination of those conditions is the key for generating smooth gait trajectories like various animal motions based on kinematic constraints and the degree of freedom.

\section{Hypothesis}

The original Theo Jansen mechanism has two static joints: one is the circle center of the input torque generator $O_{1}$ and the other is the meeting point of upper three components with two deforming rectangles and a fixed triangle $\mathrm{O}_{2}$ as shown in Figure 1 (a). Interestingly, we found a possibility of morphological changes of the gait trajectory if the second static joint $\mathrm{O}_{2}$ moves synchronously with the input circular movement around $O_{1}$ with a specific range, direction and timing difference similar like the oscillation phase difference in biological locomotive mechanism, which was previously reported by Komoda and Wagatsuma [12,13] as illustrated in Figure 1 (b). Importance of phase differences among peripheral controller (nervous system) activities that govern motion timings of body components is known for its contribution to the generation of functional movements, such as walking with leg and handling with arms, called central pattern generator (CPG) [14]. Taga [15] demonstrated that a mutual coupling of CPGs successfully provides the robotic bipedal locomotion even in unpredictable environment by changing its locomotive patterns.

In addition, similar studies have been reported to extend from such a static walking patterns to dynamics motion even with jumping [16-18]. On the other hand, the phase difference was mostly discussed in the controller design and its protocols. It is difficult to find a significant notation of the phase difference in the mechanical design with kinematics and dynamics, except the Theo Jansen mechanism [7]. The present paper addressed a constraint problem to explore possible relationships in the space spanned by axes of the amplitude, dominant direction and phase difference of $\mathrm{O}_{2}$ movement with respect to $O_{1}$ motion, under the criterion of whether or not the closed-loop linkage provides unbroken motions. For this purpose, the parametric oval orbit was used for $\mathrm{O}_{2}$ movement conventionally. We hypothesized that the modified Theo Jansen mechanism has the capability to generate multiple types of the gait trajectories under certain condition represented by a subspace of the phase-rotation-amplitude parameter space, which exhibits a significant phase relationship. The amplitude limitation is trivial in the mechanical sense yet a phase-specific motion generation was a unique fact previously unknown, which suggests that the mechanical coordination in the closed-loop linkage is consistent with schemes of biological motions that reported in experimental evidences of CPG patterns [14]. Our proposed method of step-wise

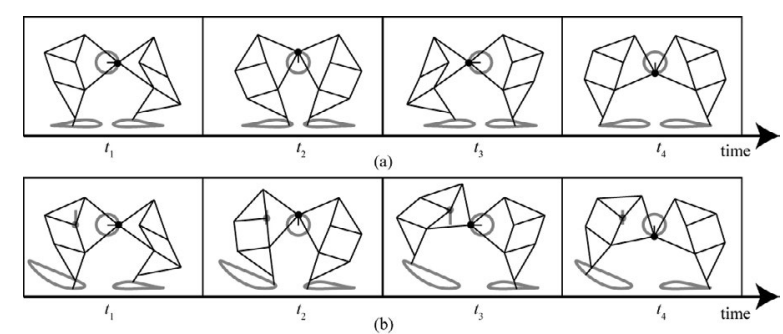

Figure 1: Locomotion behaviors generated by the Theo Jansen mechanism and the extended version. Black and gray lines respectively represent the linkages and trajectories of movable joints and end effectors as tips of legs. Postures were illustrated every $\pi / 2$ as $\mathrm{t}=\left[t_{1}, t_{2}, t_{3}, t_{4}\right]=[0, \pi / 2, \pi, 3 \pi / 2]$ defined in the driving link $O_{1}$, enabling the mechanism to go forward (leftward in the figure). (a) A temporal sequence of the walking motion of legs in the original Theo Jansen mechanism. (b) A generated motion from the extended Theo Jansen mechanism by releasing the joint lock of the joint center $\mathrm{O}_{2}$ and by adding its movement associated with $O_{1}$ movement. In this case, a climbing-like motion can be obtained in the movement of the left leg. 
determinant analyses was employed to judge whether the motion is broken or unbroken to be a functional motion and the method was validated in the following sections.

\section{Mathematical Formulations}

\section{Kinematics formulations with multibody dynamics}

In order to analyze the certain moment reaction of a constrained dynamics system, it is necessary to describe the behavior of multibody system (MBS) by using the equation of motion. The MBS is constructed by a group of rigid and flexible bodies, which depends on kinematic constraints and some force parts. Kinematic constraints demonstrate linear or quadratic dependence on the generalized Cartesian coordinate. Various approaches for the generation of the equation of motion in the MBS can be found in various ways [19-22].

If a planar mechanism is made up of $n b$ rigid bodies, the number of planar Cartesian generalized coordinates is $n c=3 n b$. The vector of generalized coordinates for the systems is written as:

$$
\boldsymbol{q}=\left[\boldsymbol{q}_{1}^{T}, \boldsymbol{q}_{2}^{T}, \cdots, \boldsymbol{q}_{n b}^{T}\right]^{T}
$$

where $\boldsymbol{q}_{i}=\left[x_{i}, y_{i}, \theta_{i}\right]_{i}^{T}$ is the vector of planar Cartesian generalized coordinates for MBS.

A kinematic constraint between body $i$ and body $j$ imposes conditions on the relative motion between the pair of bodies at arbitrary joint $k$ is obtained as:

$$
\begin{aligned}
\boldsymbol{\Phi}_{k}^{Q(i, j)} & =\left(\boldsymbol{r}_{i}+\boldsymbol{A}_{i} \boldsymbol{s}_{i}^{\prime k}\right)-\left(\boldsymbol{r}_{j}+\boldsymbol{A}_{j} \boldsymbol{s}_{j}^{\prime k}\right) \\
& =\left(\begin{array}{c}
x_{i}+x_{i}^{\prime k} \cos \theta_{i}-y_{i}^{\prime k} \sin \theta_{i} \\
-x_{j}+x_{j}^{\prime k} \cos \theta_{j}+y_{j}^{\prime k} \sin \theta_{j} \\
y_{i}+y_{i}^{\prime k} \sin \theta_{i}+y_{i}^{\prime k} \cos \theta_{i} \\
-y_{j}-x_{j}^{\prime k} \sin \theta_{j}-y_{j}^{\prime k} \cos \theta_{j}
\end{array}\right) \\
& =\mathbf{0},
\end{aligned}
$$

where $r_{i}$ is the vector to centroid of body, $A_{i}$ is the rotation transformation matrix, and $\mathrm{s}_{i}^{\prime k}$ is the local representation of body fixed vector to point $k$.

According to the configuration of the MBS defined by $n$ vector of generalized coordinates of $\mathrm{q}$ and $t$ is the time, a set of kinematic constraint equations $\phi$ are obtained as:

$$
\boldsymbol{\Phi}(\mathbf{q}, t)=\left[\begin{array}{c}
\boldsymbol{\Phi}^{K}(\mathbf{q}) \\
\boldsymbol{\Phi}^{D}(\mathbf{q}, t)
\end{array}\right]=\mathbf{0}
$$

where $\phi^{K}(\mathrm{q})$ is the kinematic constraint equation, $\phi^{D}(q, t)$ is the driving constraints of MBS.

Equation (3) is related to time to obtain the velocity constraint equation. It introduces a second differentiation of Equation (3) with respect to time to obtain the acceleration constraint equation as:

$$
\begin{aligned}
& \boldsymbol{\Phi}_{q} \dot{q}=\boldsymbol{v}, \\
& \boldsymbol{\Phi}_{q} \ddot{\boldsymbol{q}}=\boldsymbol{\gamma},
\end{aligned}
$$

where $\Phi_{\mathrm{q}}$ is the Jacobian matrix of the kinematic constraint equation, $v$ is the velocity equation and $\gamma$ is the acceleration equation.

\section{Determinant analysis for detecting non-broken motions}

Our purpose is to obtain functional motions on the extended Theo Jansen mechanism. It is important to clarify the position and posture for a specific purpose. Only if the mechanism generates a continuous orbit with the non-singular configuration throughout the orbit, we use it as the "functional orbit." The consistency between the singularity and a part of angle in the mechanism becomes an effective method to detect an irregular orbit.

The singular configurations of a general $n$-linkage will be defined for which the legs position in a certain direction cannot be realized by any finite joint position [23], which means an impossible or broken motion. Let us consider $n$ elements in the linkage and define the singular configurations using the Jacobian matrix as $\phi_{q}$. Let $n^{\prime}$ be given by :

$$
n^{\prime}=\max _{\mathbf{q}} \operatorname{rank} \boldsymbol{\Phi}_{q} \text {. }
$$

Then the singular configurations always satisfy:

$$
\operatorname{rank} \Phi_{q}<n^{\prime} \text {. }
$$

In the Theo Jansen mechanism, $n=n^{\prime} \leq 36$ and $\phi_{q}$ is square, a necessary condition for a $q$ to be singular is given by:

$$
\left|\operatorname{det} \boldsymbol{\Phi}_{q}\right|=0 \text {. }
$$

In this analysis, the singular configuration is detected by the Jacobian determinant which is zero. If the determinant is equal to zero, the orbit breaks and it exceeds the normal joint range of motion. The extended Theo Jansen mechanism has two degrees of freedom (DOF) as redundant constraints, and it is necessary to find movable ranges before the lock-up.

In the original Theo Jansen mechanism, the gait trajectory consists of motion phases including stance and swing phases, which exhibit a continuous and smooth curvature shaped like an ellipse with a sharpen edge. The morphological change of the trajectory implies a generation of other functions beyond the walking as is illustrated in Figure 2, while discussions on the functionality needs to involve the ground condition. The inevitable problem before the functionality with ground conditions is how the mechanism can be extended without the lock-up, and this paper is devoted to mathematical analyses to evaluate the end effector trajectory with respect to the parametric space to control the additional $\mathrm{O}_{2}$ movement, which is called as the extended Theo Jansen mechanism. In order to obtain relationship an original motion and the extended one, we lead to mathematical description for getting limitation of the mechanism by using determinant.

A generated mechanism is classified as following two judgment conditions as shown in Figure 3. First, a determinant checks kinematical constraints in the mechanism. If determinant is equal to zero, a generalized mechanism becomes singular configuration. Second, a certain kinematical constraint is judged for checking bifurcations in the mechanism. In quadrilateral $\mathrm{O}_{2} C E D$ in Figure 4, if angle $\mathrm{CO}_{2} \mathrm{D}$ is more than rad or if angle $\mathrm{O}_{2} \mathrm{DE}$ is more than rad, then a generalized mechanism obtains a bifurcation in this linkage. Above judgment conditions represent a broken leg's trajectory in our proposal mechanisms. Kinematics and dynamics of the original Theo Jansen mechanism and the possible extension form are clearly described through the MBD and the limitation of the mechanism is analyzed using determinant as described in the next section. 


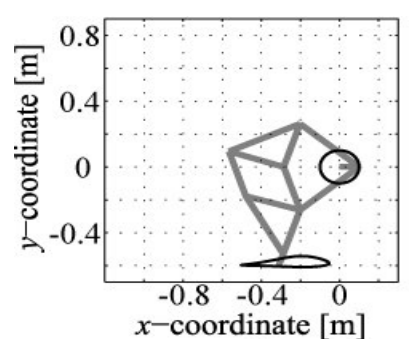

(a)

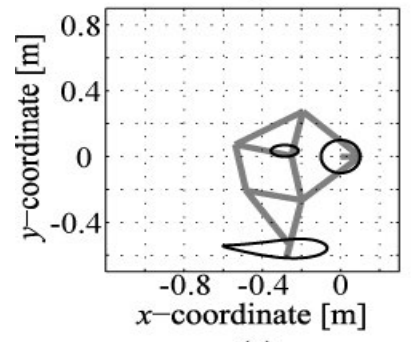

(c)

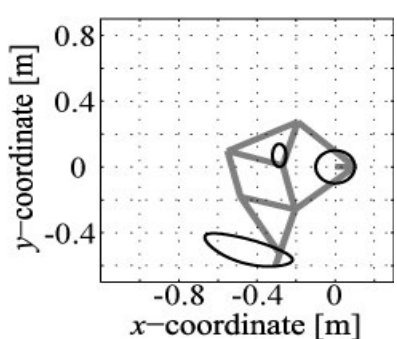

(b)

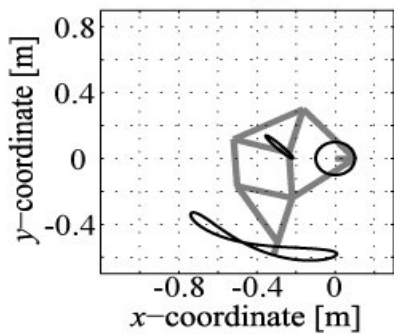

(d)
Figure 2: Examples of gait trajectories generated from the extended Theo Jansen mechanism. (a) The gait trajectory without any movement in the joint center $\mathrm{O}_{2}$ which was consistent with the one from the original mechanism. (b) A generated trajectory of the end effector with an upward rotation angle characteristics, which was provided by the $\mathrm{O}_{2}$ elliptic orbit stretched vertically. (c) A generated trajectory of the end effector to be enlarged with a similar shape with the original one, which was provided by the $\mathrm{O}_{2}$ elliptic orbit stretched horizontally. (d) A figure-of-eight trajectory provided by the $\mathrm{O}_{2}$ elliptic orbit stretched in $3 \pi / 4$ rad direction. $O_{1}$ and $O_{2}$ rotated synchronously from the same angle.

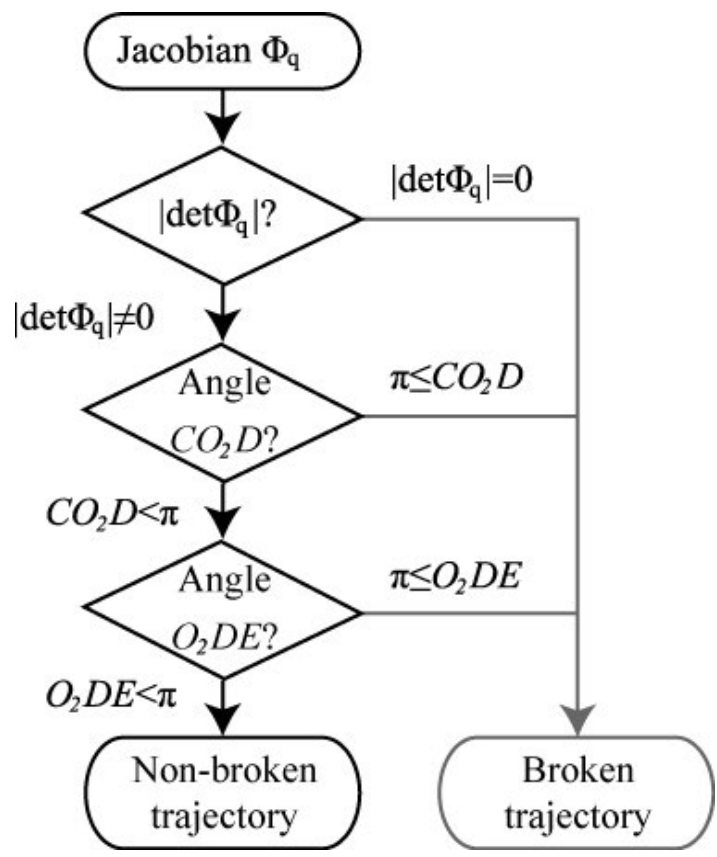

Figure 3: Procedures to detect a singular configuration by using the determinant and target angles in the mechanism. The first criterion is whether or not the determinant $\left|\operatorname{det} \Phi_{\mathrm{q}}\right|$ is equal to zero, and the second and third criterions are to evaluate whether angles of $\mathrm{CO}_{2} \mathrm{D}$ and $\mathrm{O}_{2} \mathrm{DE}$ located on the underside of $\mathrm{O}_{2}$ exceeds $\pi$ or not. The set of criterions can be used for the deadlock check.

\section{Extension Model of the Theo Jansen Mechanism}

According to the common framework of the model definition and preliminaries in section of "Mathematical Formulations," a closedloop linkage, the Theo Jansen mechanism can be treated consistently in multibody dynamics (MBD) methods, which means that a legged robot is constructed by individual differential algebraic equations to be able to analyze the placement, velocity, acceleration, and determinant.

Original Theo Jansen mechanism: The mathematical model of the Theo Jansen mechanism is described according to the kinematics formulation in the previous section. As is illustrated in Figure 4, vectors q with 36 elements including placements and attitude angles are represented in generalized coordinates:

$$
\boldsymbol{q}=\left[\boldsymbol{q}_{1}^{T}, \boldsymbol{q}_{2}^{T}, \boldsymbol{q}_{3}^{T}, \boldsymbol{q}_{4}^{T}, \boldsymbol{q}_{5}^{T}, \boldsymbol{q}_{6}^{T}, \boldsymbol{q}_{7}^{T}, \boldsymbol{q}_{8}^{T}, \boldsymbol{q}_{9}^{T}, \boldsymbol{q}_{10}^{T}, \boldsymbol{q}_{11}^{T}, \boldsymbol{q}_{12}^{T}\right]^{T} .
$$

A set of kinematic constraint equations $\phi$ are given by Equation (3). The first thirty-five elements of the column matrix $\phi^{K}(\mathrm{q})$ are derived from kinematic constraint equa-tions. Whereas the last element $\phi^{D}(\mathrm{q}, t)$ is derived by driving constraint equation, the equation of kinematic constraints and a driving constraint are obtained as:

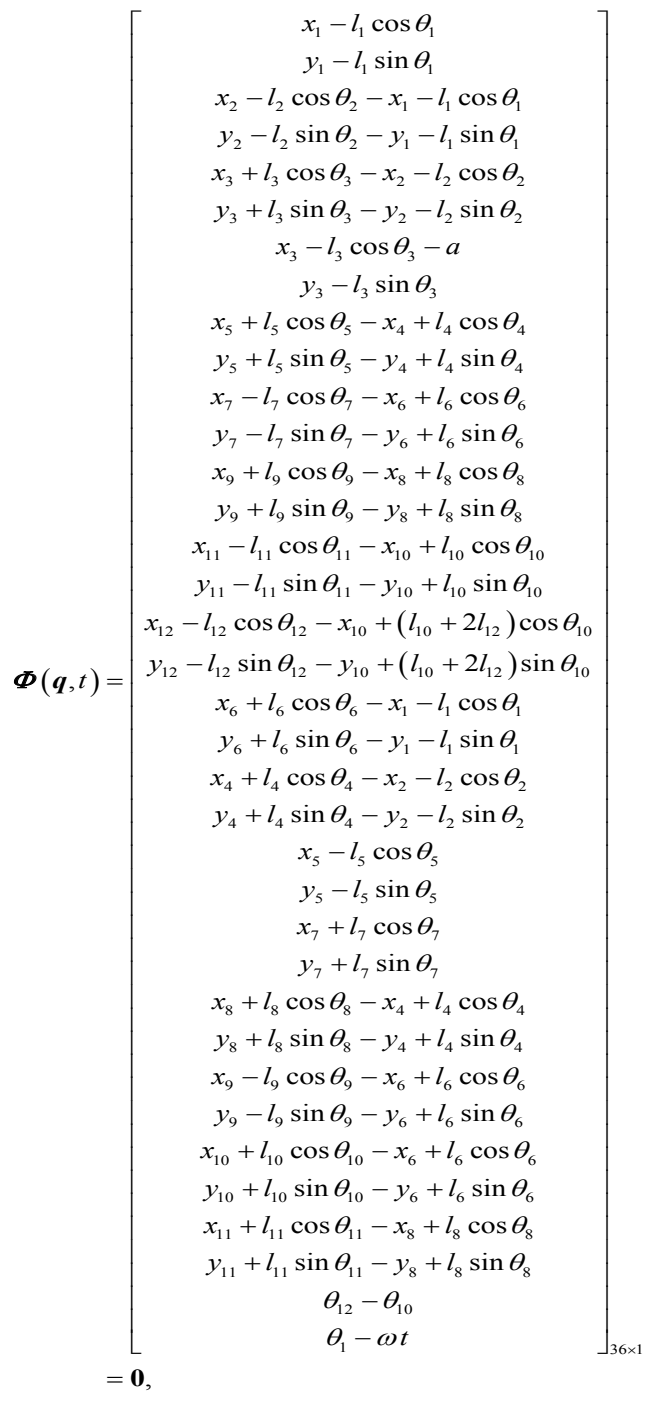


where $l_{1}$ to $l_{12}$ are link lengths, $t$ is time, and $\omega$ is angular velocity of the driving link in the mechanism. Originally the Theo Jansen mechanism is known as eleven linkages, while in this case it is described twelve linkages in the MBD with generalized coordinates. Table 1 denotes a set of parameter values of the Theo Jansen mechanism. Therefore, the Jacobian matrix $\phi_{q}$ is obtained as:

$$
\boldsymbol{\Phi}_{q}=\left[\frac{\partial \boldsymbol{\Phi}(\boldsymbol{q}, t)}{\partial \boldsymbol{q}}\right]_{36 \times 36},
$$

where it allows us to investigate placement, velocity and acceleration analyses kinematically.

Extension with parametric ovals: A few DOF are one good advantage obtained from the parallel linkage mechanism for generating a designed motion. According to our hypothesis, the mechanism can be modified by changing the static joint of $\mathrm{O}_{2}$ to the movable one, abbreviated as "joint center", reminiscent of the knee point of the human leg. It is a partial extension of the DOF against the original mechanism toward additional motion generations, and the new motion to the joint center $\left(\mathrm{O}_{2}\right)$ in Figure 4 is given as follows:

$$
\begin{aligned}
& {\boldsymbol{s}_{O_{1}}^{\prime}}_{O_{1}}^{O}=\left[\begin{array}{ll}
0 & 0
\end{array}\right]^{T} \text {, } \\
& \boldsymbol{s}_{O_{2}}{ }^{O_{1}}=\left[\begin{array}{ll}
-a & 0
\end{array}\right]^{T} \text {. }
\end{aligned}
$$

Let $A_{x}, A_{y}, \omega, t, \delta_{x}, \delta_{y}, b_{x}$, and $b_{y}$ be amplitude, angular velocity, time, phase difference, and axis shift in $x$ and $y$ coordinates, respectively:

$$
\begin{aligned}
& x_{O_{2}}(t)=A_{x} \cos \left(\omega t-\delta_{x}\right)+b_{x}, \\
& y_{O_{2}}(t)=A_{y} \sin \left(\omega t-\delta_{y}\right)+b_{y} .
\end{aligned}
$$

Equation (14) and Equation (15) describe an orbital shape in the leg. These equations, which have seven conditions for detecting one exact motion, are for general condition in the extended Theo Jansen mechanism. In the previous research, we found a functional leg's orbit under a limited condition [12]. The phase difference between $\mathrm{O}_{2}$ and $O_{1}$ and a vertical mobility of $O_{2}$ were considered to be the clues for obtaining additional motions in the extended mechanism. In order to observe functional leg's trajectory, the number of variables in Equation (14) and Equation (15) can be reduced by its dependency. By assuming that $\delta_{x}$ and $\delta_{y}$ are equal to the single variable $\delta$, the equivalent phase difference is given by Equation (14) and Equation (15) in the condition that $b_{y}$ is equal to $A_{y}$ and $b_{x}$ is equal to zero. If $b$ is less than $A_{y}$, the toe position exceeds the level of the ground, which means digging of the

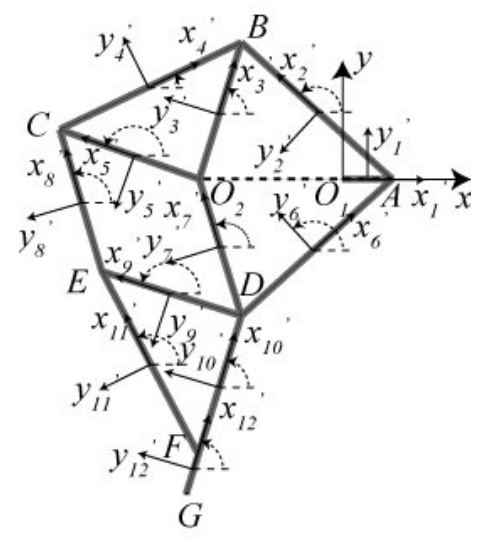

Figure 4: Generalized coordinates on the Theo Jansen mechanism. This figure was plotted in $x$ and $y$ coordinate axes, other than rotation angle based descriptions of each joints [13].

\begin{tabular}{|c|c|c|}
\hline Parameter & Sides & Length $\left[\times \mathbf{1 0}^{-3} \mathbf{~ m}\right]$ \\
\hline$I_{1}$ & $O_{1} A$ & 50.0 \\
\hline$I_{2}$ & $A B$ & 200.0 \\
\hline$I_{3}$ & $O_{2} B$ & 137.5 \\
\hline$I_{4}$ & $B C$ & 200.0 \\
\hline$I_{5}$ & $O_{2} C$ & 147.5 \\
\hline$I_{6}$ & $A D$ & 200.0 \\
\hline$I_{7}$ & $O_{2} D$ & 137.5 \\
\hline$I_{8}$ & $C E$ & 142.5 \\
\hline$I_{9}$ & $D E$ & 145.0 \\
\hline$I_{10}$ & $D F$ & 140.0 \\
\hline$I_{11}$ & $E F$ & 200.0 \\
\hline$I_{12}$ & $F G$ & 40.0 \\
\hline$a$ & $O_{1} O_{2}$ & 143.0 \\
\hline
\end{tabular}

Table 1: Parameters of link length on the Theo Jansen mechanism.

ground. Thus, we modified Equation (14) and Equation (15) as follows:

$$
\begin{aligned}
& x_{O_{2}}(t)=A_{x} \cos (\omega t-\delta), \\
& y_{O_{2}}(t)=A_{y}[\sin (\omega t-\delta)+1] .
\end{aligned}
$$

The rotation matrix is used to change the rotation angle of the parametric orbit of $\mathrm{O}_{2}$ (the vertical longitudinal ellipse is assigned as 0 $[\mathrm{rad}])$ is given as:

$$
\begin{aligned}
\left(\begin{array}{l}
x_{O_{2}}(t) \\
y_{O_{2}}(t)
\end{array}\right) & =\left(\begin{array}{cc}
\cos \phi & -\sin \phi \\
\sin \phi & \cos \phi
\end{array}\right)\left(\begin{array}{c}
A_{x} \cos (\omega t-\delta) \\
A_{y}[\sin (\omega t-\delta)+1]
\end{array}\right) \\
& =\left(\begin{array}{l}
A_{x} \cos \phi \cos (\omega t-\delta)-A_{y} \sin \phi[\sin (\omega t-\delta)+1] \\
A_{x} \sin \phi \cos (\omega t-\delta)+A_{y} \cos \phi[\sin (\omega t-\delta)+1]
\end{array}\right),
\end{aligned}
$$

where, $\phi$ is an arbitrary angle of the parametric oval. Furthermore, we added following two conditions for keeping a certain placement in $\mathrm{O}_{2}$. First, minimum $\mathrm{O}_{2}$ orbit point in $y$ mechanism Coordinate placement is zero. Second, cross point between long axis and short axis in ellipse orbit $\mathrm{O}_{2}$ is zero in $x$ coordinate. We expect that these conditions can assure the extended mechanism to prevent drilling in the ground by its legs. Thus, equation (18) is modified as following:

$$
\left(\begin{array}{l}
x_{O_{2}}(t) \\
y_{O_{2}}(t)
\end{array}\right)=\left(\begin{array}{c}
x_{O_{2}}(t)-0.5\left(\min \left\{x_{O_{2}}(t)\right\}+\max \left\{x_{O_{2}}(t)\right\}\right) \\
y_{O_{2}}(t)+\left|\min \left\{y_{O_{2}}(t)\right\}\right|
\end{array}\right) .
$$

Therefore, the relative coordinates drives are as follows, finally, the relative coordinates drive are given as follows:

$$
\begin{aligned}
& \boldsymbol{\Phi}_{O_{2}}^{Q(3)}(\boldsymbol{q}, t)=\left(\begin{array}{c}
x_{3}-l_{3} \cos \theta_{3}+a-x_{O_{2}}(t) \\
y_{3}-l_{3} \sin \theta_{3}-y_{O_{2}}(t)
\end{array}\right)=\mathbf{0}, \\
& \boldsymbol{\Phi}_{O_{2}}^{Q(5)}(\boldsymbol{q}, t)=\left(\begin{array}{c}
x_{5}-l_{5} \cos \theta_{5}+a-x_{O_{2}}(t) \\
y_{5}-l_{5} \sin \theta_{5}-y_{O_{2}}(t)
\end{array}\right)=\mathbf{0}, \\
& \boldsymbol{\Phi}_{O_{2}}^{Q(7)}(\boldsymbol{q}, t)=\left(\begin{array}{c}
x_{7}-l_{7} \cos \theta_{7}+a-x_{O_{2}}(t) \\
y_{7}-l_{7} \sin \theta_{7}-y_{O_{2}}(t)
\end{array}\right)=\mathbf{0} .
\end{aligned}
$$

According to the above modification, the extended Theo Jansen mechanism has two degrees of freedom as redundant constraints. The mechanism can be investigated by changing four parameters $A_{x}, A_{y} \phi$, and $\delta$ to reveal what kinds motions are generated.

\section{Results of Numerical Simulations}

In this section, results of numerical simulations were presented as a consequence of the modeling discussed Section of "Extension 
Model of the Theo Jansen Mechanism." As hypothesized in Section of "Hypothesis," extended functional motions are able to be generated by adding a synchronous movement of $\mathrm{O}_{2}$ joint center, which represents a kind of knee position as an analogy so that the driving cycle of $O_{1}$ and the end effector motion of $G$ are assumed to be inguinal and toe, respectively [12]. For detailed analyses of such extended functional motions, the parametric oval movement of $\mathrm{O}_{2}$ was proposed as formulated in Section of "Extension with Parametric Ovals" and this section is devoted to the detailed examination of placement, posture, velocity, acceleration, and determinant of the extended Theo Jansen mechanism. Parameters and conditions for the analyses were described in Table 2, which is used in MATLAB based numerical simulation with the Newton-Raphson method. In following analyses, the constant angular velocity $\omega=0.5 \pi \mathrm{rad} / \mathrm{s}(15 \mathrm{rpm})$ was assumed to be the driving link rotation of $O_{1}$ consistently for every numerical simulation, as shown in Figure 4 consistently for every numerical simulations. In placement, curvature, velocity, acceleration and determinant analyses, the end effector, $G$ which is the tip of the leg, is analyzed by using the generalized placement of the grounding link, and then the end effector placement of the Theo Jansen mechanism $G=\left[G_{x}, G_{y}\right]$ was calculated by the center of twelve links $\left[x_{12}, y_{12}\right]$. In following subsections, prominent three motions were analyzed as functional movements for various locomotive actions especially for uneven fields.

\section{Characteristic analyses on the extended Theo Jansen mechanism}

Climbing-like motion: As an example of generated trajectory in the extended Theo Jansen mechanism using a set of parameters in the first column of Table 2, the temporal sequence of placements and postures during a cycle was obtained (Figure 5 (a)). The driving link input was given as the circular trajectory with the constant angular velocity $\omega$ (the circle at the origin of the figure) which is finally transformed into an elliptic trajectory as the leg's trajectory including swing and stance phases of the gait. Since highest and lowest placements of the trajectory were representative points of swing and stance phases, the leg's height was obtained by the subtraction of the minimum placement $-0.61 \mathrm{~m}$ from the maximum placement $-0.05 \mathrm{~m}$ to be $0.56 \mathrm{~m}$ from the ground. Thus, the generated trajectory was eight times higher than the height of the original gait trajectory and was $64.4 \%$ of the system's total height (Figure 5 (b)). For the definition of the 'climbing' ability, gradeability should be considered as the maximum slope that a vehicle can climb in a stable condition, preventing slipping of wheels and legs on the ground and rollover that happens if the center of mass of the vehicle exceeds an acceptable range of the backward inclining, which is calculated by the line connecting of two peak points of the curvature (Figure 5 (c)).

The curvature is defined by using an edge of trajectory as $\kappa(t)=\frac{\dot{x} \ddot{y}-\ddot{x} \dot{y}}{\left(\dot{x}^{2}+\dot{y}^{2}\right)^{3 / 2}}$, where $x$ and $y$ are placements of the leg's trajectory. In the assumption that the friction of legs on the ground is high enough

\begin{tabular}{|l|l|l|l|}
\hline Parameter & Climbing-like & Figure-of-eight & Dropping-down \\
\hline$A_{\mathrm{x}}\left[\times 10^{-3} \mathrm{~m}\right]$ & 70 & 20 & 100 \\
\hline$A_{\mathrm{y}}\left[\times 10^{-3} \mathrm{~m}\right]$ & 140 & 100 & 70 \\
\hline$\delta[\mathrm{rad}]$ & $-270 \pi / 180$ & $-240 \pi / 180$ & $-30 \pi / 180$ \\
\hline$\phi[\mathrm{rad}]$ & 0 & $45 \pi / 180$ & 0 \\
\hline
\end{tabular}

Table 2: Parameters for generating three functional motions. $A_{x}$ and $A_{y}$ denote respectively horizontal and vertical amplitudes of the $\mathrm{O}_{2}$ orbit, $\delta$ is the phase difference between the $O_{2}$ and $O_{1}$ movements, $\phi$ rad is the rotation angle of Elliptic orbit of $O_{2}$, and $\omega$ Was given by $\pi / 2 \mathrm{rad} / \mathrm{s}$ consistently. During the one cycle to take $t=0 \leq t \leq 4 \mathrm{~s}$ with the time step $d t=1 / 90 \mathrm{~s}$ using the ODE solver as Newton-Raphson method.

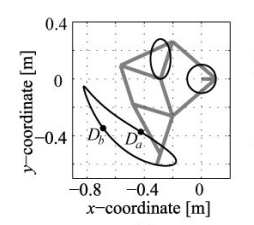

(a)
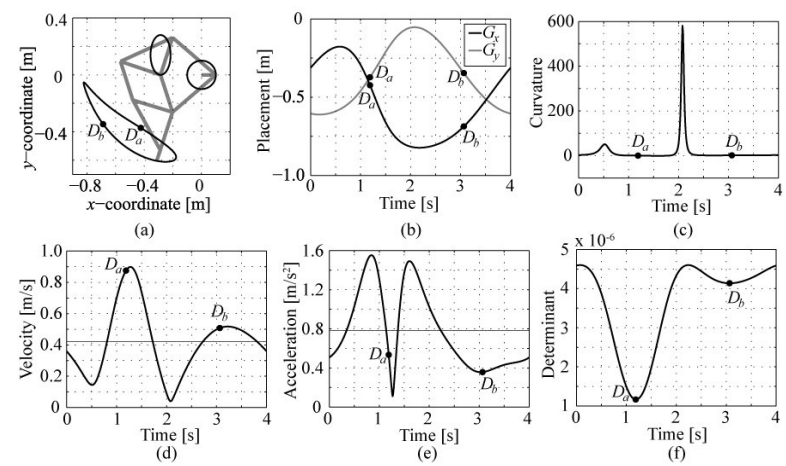

Figure 5: Detail analyses using multibody dynamics in the case of climbing like motion. The generated trajectory associated with the $\mathrm{O}_{2}$ movement was shown in (a), temporal sequences of placements of $G_{x}$ and $G_{y}$ as the end effector movement was in (b), the curvature of the trajectory given by $\frac{\dot{x} \ddot{y}-\ddot{y} \dot{y}}{\left(\dot{x}^{2}+\dot{y}^{2}\right)^{3 / 2}}$

was is (c), the velocity (d) and acceleration (e) of the end effector were shown respectively. Temporal sequences of determinant was shown in (d). $D_{a}$ and $D_{b}$ were obtained as local minimums of the determinant and they were superimposed each analysis. According to the analyses, local minimums of the determinant could not simply be correlated with peak and valley each plot. In velocity and acceleration analyses, individual average values were shown as gray lines.

for stable onward movements, the most important issue is the acceptable range of the backward inclining, which is calculated by the relationship between the position of the center of mass and the endpoint of the contact area with the ground. In the case of legged robots, the contact area can be given by the gait trajectory. Ghassaei [24] investigated the position of the center of mass in the Theo Jansen mechanism through the integration of individual link analyses and concluded that it was located at just below the lower end of the driving link. According to the result, the maximum rotation angle is able to incline backward without rollover was about $45^{\circ}$ and it can be considered as the acceptable range of the backward inclining in the Theo Jansen mechanism. The climbing-like motion of our result allows legs to lift up to $39.5^{\circ}$ within the acceptable range, which is calculated by the slope of the line connecting inflection points forward and backward on the gait trajectory. According to the review of Seeni [25], wheel based robots can climb up to $25^{\circ}$, legged robots such as 8-legged system SCORPION [26] and 6-legged DLR Walker robot [27], can be estimate to be able to climb up to $35^{\circ}$ and wheel-leg hybrid robots can climb higher than $35^{\circ}$. Hopping robots or hoppers are obviously suitable machine for climbing and hop-roll hybrids have wheels and legs in upper and lower surfaces to provide high capabilities beyond the concept of rollover accidents of vehicles. In comparison with those capabilities, our extended Theo Jansen has a climbing capability higher than wheel based robots and usual legged robots, and close to the wheel-leg hybrid robots $[28,29]$.

According to the velocity analysis of the leg as shown in Figure 5 (d), minimum and maximum velocities were $0.04 \mathrm{~m} / \mathrm{s}$ and $0.90 \mathrm{~m} / \mathrm{s}$, respectively and then the mean velocity was $0.42 \mathrm{~m} / \mathrm{s}$. The first peak appeared at the maximum speed of $0.90 \mathrm{~m} / \mathrm{s}(t=1.278 \mathrm{~s})$, the second peak appeared at the point with $0.52 \mathrm{~m} / \mathrm{s}$ speed $(t=3.222 \mathrm{~s})$. As plotted on the trajectory, the first peak point was located at the midpoint in the swing phase and the second peak was located in the swing phase after passing through the highest placement of the trajectory. The trajectory shaped like a climbing-like motion for climbing obstacle and the leg moved quickly in the swing phase and slowly in the stance phase.

In the acceleration analysis in Figure 5 (e), there were two peaks at 
$1.55 \mathrm{~m} / \mathrm{s}^{2}(t=0.844 \mathrm{~s})$ and $1.49 \mathrm{~m} / \mathrm{s}^{2}(t=1.600 \mathrm{~s})$ in the plot of the temporal sequence of the absolute acceleration value of the leg movement. The two points were located respectively at the moment just after the lifting up the leg as an initiation of the swing phase and the moment just before the transition of the moving direction, i.e., the curvature peak, in the swing phase. This result indicate that the mechanism moves fast when the leg takes off from the ground accompanied with a complex acceleration control by maximizing the acceleration at the midpoint of the swing phase and moves slow by minimizing the speed at the curvature peak point and then touches the ground by decreasing the acceleration to form the smooth trajectory.

In the determinant analysis as shown in Figure 5 (f), minimum and maximum determinants of the system were obtained as $1.16 \times 10^{-6}$ and $4.60 \times 10^{-6}$, respectively. When an angle was $107 \pi / 180 \mathrm{rad}$, a mechanism approached to a singularity posture. In this condition, a leg is moving swing phase for reaching edge in the trajectory. Local minimums of the determinant $D_{a}$ and $D_{b}$ during a cycle could not simply be correlated with characteristic points in the placement, curvature, velocity and acceleration.

Figure-of-eight motion: As a further example of the generated trajectory in the extended Theo Jansen mechanism, a figure-of-eight end effector trajectory was obtained using a set of parameters in the second column of Table 2, as shown in Figure 6 (a). Figure-of-eight trajectories were known to exhibit biological motions, such as swimming $[30,31]$ and flying [32-34] even in microorganism [35]. Insect motions in flight realize smooth movements with a less energy consumption [32,33], which are easily generated by a combination of oscillatory components with amplitude and phase modulations known as Lissajous figures [36]. Therefore, mechanisms to generate such trajectories have been investigated in bio-inspired robotics [37-41]. On the other hand, figureof-eight trajectories were not recognized in the human locomotion before, while recent advance of three dimensional human motion analyses revealed the existence of figure-of-eight trajectories in gait if it is drawn to designate the root of the thigh as the origin [42].

In fact, the human locomotion generated by a complex human musculoskeletal system is not simply equivalent to the insect motion generation. Interestingly, as Ivanenko [43] demonstrated, there exists a consistency of modular based control mechanism to reduce the freedom of degrees using a synchronous coordination among modular units, which generates a movement-speed dependent change in the gait trajectory. They classified gait trajectories into walking, running, hopping, crouched walking, obstacle avoidance and air-stepping, and found a figure-of-eight trajectory in the case of running.

In the present study, the height of the figure-of-eight trajectory was $0.25 \mathrm{~m}$ from the ground (29.6\% against the total height), which suggested a non-trivial relationship with the human locomotion.

According to the velocity analysis of the leg as shown in Figure 6 (d), minimum and maximum velocities were $0.04 \mathrm{~m} / \mathrm{s}$ and $0.57 \mathrm{~m} / \mathrm{s}$, respectively and then the mean velocity was obtained as $0.30 \mathrm{~m} / \mathrm{s}$. The first peak appeared at the maximum speed of $0.57 \mathrm{~m} / \mathrm{s}(t=1.556 \mathrm{~s})$, the second peak appeared at the point with $0.42 \mathrm{~m} / \mathrm{s}(t=3.633 \mathrm{~s})$. As shown by the plot of trajectory, the first point is located at the midpoint in the swing phase and the second peak was located at the midpoint in the swing phase after passing through the highest placement of the trajectory. The trajectory shaped like a figure-of-eight motion for running slowly. In addition, the leg moved quickly in the swinging up motion and slowly in the swinging back motion.

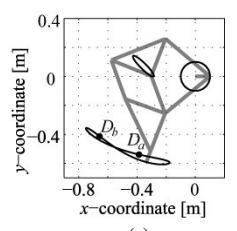

(a)

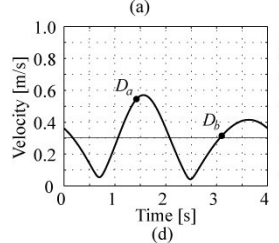

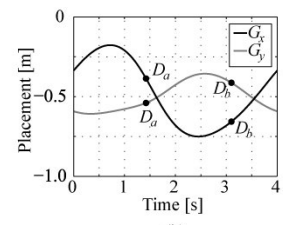

(b)

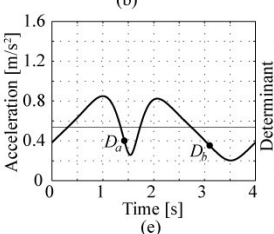

(e)
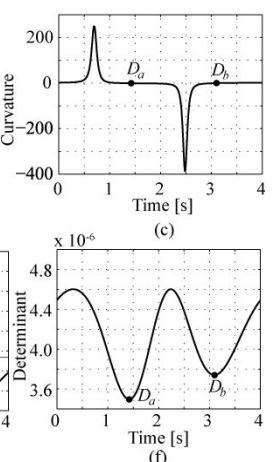

Figure 6: Detail analyses using multibody dynamics in the case of figureof-eight motion. The generated trajectory associated with the $\mathrm{O}_{2}$ movement was shown in (a), temporal sequences of placements of $G_{x}$ and $G_{y}$ as the end effector movement was in (b), the curvature of the trajectory given by $\frac{\dot{x} \ddot{y}-\ddot{x} \dot{y}}{\left(\dot{x}^{2}+\dot{y}^{2}\right)^{3 / 2}}$ was in (c), the velocity (d) and acceleration (e) of the end effector, with average values (gray line) were shown respectively. Temporal sequences of determinant was shown in (d). $D_{a}$ and $D_{b}$ were obtained as local minimums of the determinant and they were superimposed each analysis. $D$ was larger than Figure 5.

In the acceleration analysis in Figure $6(\mathrm{e})$, there were two peaks of points $0.85 \mathrm{~m} / \mathrm{s}^{2}(t=1.000 \mathrm{~s})$ and $0.82 \mathrm{~m} / \mathrm{s}^{2}(t=2.067 \mathrm{~s})$, having a similar value. In the determinant analysis as shown in Figure 6 (f), minimum and maximum determinants of the system were obtained as $3.50 \times 10^{-6}$ and $4.60 \times 10^{-6}$, respectively. The minimum determinant was obtained when the angle was $128 \pi / 180 \mathrm{rad}$, while it was far from a singularity posture as values larger than $3.4 \times 10^{-6}$.

Dropping-down motion: As the final example, a dropping-down motion was generated via the extended Theo Jansen mechanism using a set of parameters in the last column of Table 2, and placements and postures during a cycle were illustrated in Figure 7 (a). It is difficult to find a simple coincidence between the dropping-down motion with biological motions to realize a specific function, while it should be focused on the compensation property. The generated motion exhibiting as a small circular orbit was obtained by the cancellation of two types of motions, so that the joint center $\mathrm{O}_{2}$ moves to offset the driving link cyclic motion $O$ inside the mechanism by anti-phase cyclic motion between two cycles. Indeed, the trajectory height was reduced as $0.13 \mathrm{~m}$ from the ground ( $15.6 \%$ of the total height).

According to the velocity analysis (Figure 7 (d)), minimum and maximum velocities were $0.004 \mathrm{~m} / \mathrm{s}$ and $0.13 \mathrm{~m} / \mathrm{s}$ (the mean velocity was $0.08 \mathrm{~m} / \mathrm{s}$ ), which were quite small in comparison with above two conditions. The first peak was $0.90 \mathrm{~m} / \mathrm{s}(t=1.278 \mathrm{~s})$, and the second peak was $0.52 \mathrm{~m} / \mathrm{s}(t=3.222 \mathrm{~s})$. In the acceleration analysis (Figure $7(\mathrm{e}))$, a similar tendency appeared as small values with three peaks as $0.18 \mathrm{~m} / \mathrm{s}^{2}$ $(t=0.522 \mathrm{~s}), 0.22 \mathrm{~m} / \mathrm{s}^{2}(t=2.233 \mathrm{~s})$ and $0.20 \mathrm{~m} / \mathrm{s}^{2}(t=2.756 \mathrm{~s})$.

In the determinant analysis (Figure 7 (f)), minimum and maximum determinants of the system were obtained as $2.42 \times 10^{-6}$ and $4.39 \times$ $10^{-6}$ respectively. When the angle was $235 \pi / 180 \mathrm{rad}$, the minimum determinant was obtained, which was not critical and apart from a singularity posture.

In Figure 8, characteristic properties of the acceleration during a cycle were analyzed in comparison of the three generated trajectories obtained from the extended Theo Jansen mechanism and an original trajectory. The line width represented the amount of the acceleration, which is proportional to the force generation. By using the parametric 
orbit defined in $\mathrm{O}_{2}$, the original gait trajectory (Figure 8 (a)) was successfully modified to a climbing-like motion (Figure 8 (b)), a figureof-eight motion (Figure 8 (c)) and a dropping-down or less mobility motion (Figure $8(\mathrm{~d})$ ). As represented as the line width, a consistent acceleration change was found in Figure 8 (a)-8 (c) as the twisted shape, which included a sharp negative peak of the acceleration change. Such an acceleration property acted as the quick take-off from the stance phase to the swing phase and the soft grounding from the swing phase to the stance phase, which realized a smooth gait trajectory. In all three cases, the minimum determinant marked as $D_{a}$ coincidentally appeared just before the negative peak. The result showed that the consistency through the trajectory modification associated with the $\mathrm{O}_{2}$ movement preserved the properties for generating a smooth gait trajectory even in modified ones.

\section{Functional trajectory generation focusing on amplitude change}

In the previous section, contribution of the additional movement in the joint center $\mathbf{O}_{2}=\left[x_{\mathrm{O}_{2}}, y_{\mathrm{O}_{2}}\right]$ for modifying the trajectory of the end effector was demonstrated and some functional motions were found based on the systematic parametric change. On the other hand, analysis of the robustness to pre-vent the generation of broken motion or dead lock is necessary in accordance with the criterion defined in

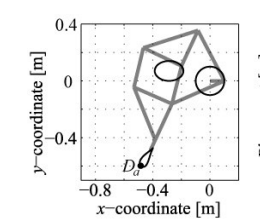

(a)

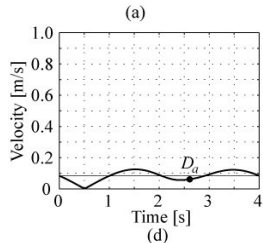

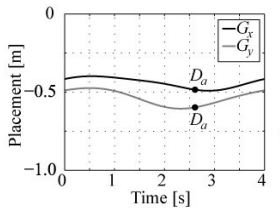

(b)

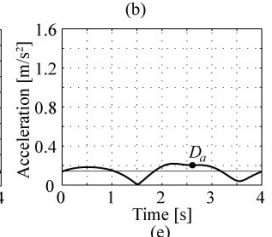

(e)
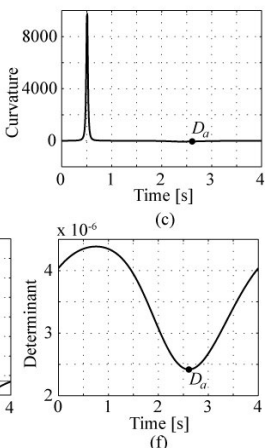

Figure 7: Detail analyses using multibody dynamics in the case of figureof-eight motion. The generated trajectory associated with the $\mathrm{O}_{2}$ movement was shown in (a), temporal sequences of placements of $G_{x}$ and $G_{y}$ as the end effector movement was in (b), the curvature of the trajectory given by $\frac{x \ddot{y}-\ddot{x} \dot{y}}{\left(\dot{x}^{2}+\dot{y}^{3}\right)^{3 / 2}}$ was in (c), the velocity (d) and acceleration (e) of the end effector,

with average values (grey line) were shown respectively. Temporal sequences of determinant was shown in (d). In this case, a single local minimum of the determinant was found as $D$, and velocity and acceleration were small in the whole period, which represent that the $O_{1}$ movement was counterbalanced by the $\mathrm{O}_{2}$ movement.

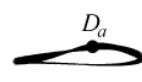

(a)

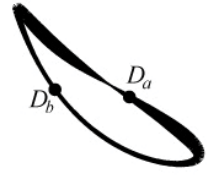

(b)

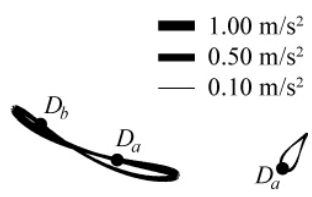

(c) (d)
Figure 8: Comparison among four types of generated gait trajectories with acceleration changes of the leg. (a) The original trajectory, (b) climbing like motion obtained in Figure 5, (c) figure-of-eight motion obtained in Figure 6, (d) dropping-down motion obtained in Figure 7. As a common property in (a)-(c), the local minimum of the determinant $D$ appeared at the point just before the local minimum of the acceleration (transition between speeding up and down) in the swing phase.
Section of "Determinant Analysis for Detecting Non-Broken Motions." In this section, characteristic analysis of the generated trajectory was investigated by focusing on amplitudes of the parametric orbit $\mathrm{O}_{2}$. According to Equation (16) and Equation (17), a set of four parameters that consist of horizontal and vertical amplitudes $A_{x}$ and $A_{y}$, the rotation angle applied to the $\mathrm{O}_{2}$ orbit as $\phi$, and the phase difference determining what kind of the new trajectory is generated. In this section, generated trajectories were analyzed by focusing on the amplitudes $A_{x}$ and $A_{y}$ from 0 to $350 \times 10^{-3} \mathrm{~m}$ using parameters of Table 3 .

In the case of the $A_{x}$ variation, various trajectories were examined with the horizontal amplitude $A_{x}$ changing from 0 to $350 \times 10^{-3} \mathrm{~m}$ associated with the change of the rotation angle $\phi$ under the fixed condition of the vertical amplitude $A_{y}=100 \times 10^{-3} \mathrm{~m}$. Figure 9 (a) illustrated 96 types of the $O_{2}$ parametric orbit by the combination of eight amplitudes of $A_{x}$ and twelve rotation angles, which were plotted in concentric circles with respect to those variations, and Figure 9 (b) showed resultant trajectories of the end effector in the same arrangement. Black dots showed a starting point in the joint center $\mathrm{O}_{2}$ moving in the anti-clockwise direction. Generated trajectories included oval, halfmoon, and figure-of-eight non-broken shapes. Broken trajectories were plotted as gray lines and serious broken trajectories were omitted from plot (the movement exceeded over $1 \mathrm{~m}^{2}$ area). The serious broken types were denoted by cross and star marks after the classification using the criterion defined in Section of "Determinant Analysis for Detecting Non-Broken Motions" and illustrated in Figure 3 (the cross and star marks represent respectively the determinant condition failure and angle condition failure). Fine trajectories of 34 types were obtained and the success rate was $35.42 \%$. According to the change of the horizontal amplitude $A_{x}$, generated trajectories varied from small shapes to large shapes. Non-broken trajectories $A$ obtained was from 0 to $250 \times 10^{-3} \mathrm{~m}$, when $\phi$ was equal to $\pi / 6$ and $2 \pi / 6 \mathrm{rad}$. According to the change of $\phi$, generated trajectories were demonstrated in a certain angle range as $-2 \pi / 6 \leq \phi \leq 2 \pi / 6 \mathrm{rad}$. On the other hands, broken trajectories were generated when angle ranges as $\pi / 2 \leq \phi \leq$ $7 \pi / 6 \mathrm{rad}$.

In the case of the $A_{y}$ variation, various trajectories were examined

\begin{tabular}{|c|c|c|}
\hline Parameter & $\boldsymbol{A}_{x}$ variation & $\boldsymbol{A}_{y}$ variation \\
\hline$A_{x}\left[\times 10^{-3} \mathrm{~m}\right]$ & $0 \leq A_{x} \leq 350$ & 100 \\
\hline$A_{y}\left[\times 10^{-3} \mathrm{~m}\right]$ & 100 & $0 \leq A_{y} \leq 350$ \\
\hline$\omega[\mathrm{rad} / \mathrm{s}]$ & $\pi / 2$ & $\pi / 2$ \\
\hline$t[\mathrm{~s}]$ & $0 \leq t \leq 4$ & $0 \leq t \leq 4$ \\
\hline$d t[\mathrm{~s}]$ & $1 / 90$ & $1 / 90$ \\
\hline$\delta[\mathrm{rad}]$ & 0 & 0 \\
\hline$\phi[\mathrm{rad}]$ & $0 \leq \phi \leq 11 \pi / 6$ & $0 \leq \phi \leq 11 \pi / 6$ \\
\hline$d \phi[\mathrm{rad}]$ & $\pi / 6$ & $\pi / 6$ \\
\hline
\end{tabular}

Table 3: Parameters for generating functional trajectories in amplitude changing.

\begin{tabular}{|c|c|c|c|}
\hline Parameter & Sample & Tangential ellipse & Normal ellipse \\
\hline$A_{x}\left[\times 10^{-3} \mathrm{~m}\right]$ & 100 & 50 & 100 \\
\hline$A_{y}\left[\times 10^{-3} \mathrm{~m}\right]$ & 100 & 100 & 50 \\
\hline$\omega[\mathrm{rad} / \mathrm{s}]$ & $\pi / 2$ & $\pi / 2$ & $\pi / 2$ \\
\hline$t[\mathrm{~s}]$ & $0 \leq t \leq 4$ & $0 \leq t \leq 4$ & $0 \leq t \leq 4$ \\
\hline$d t[\mathrm{~s}]$ & $1 / 90$ & $1 / 90$ & $1 / 90$ \\
\hline$\delta[\mathrm{rad}]$ & $0 \leq \delta \leq 11 \pi / 6$ & $0 \leq \delta \leq 11 \pi / 6$ & $0 \leq \delta \leq 11 \pi / 6$ \\
\hline$d \delta[\mathrm{rad}]$ & $\pi / 6$ & $\pi / 6$ & $\pi / 6$ \\
\hline$\phi[\mathrm{rad}]$ & $0 \leq \phi \leq 11 \pi / 6$ & $0 \leq \phi \leq 11 \pi / 6$ & $0 \leq \phi \leq 11 \pi / 6$ \\
\hline$d \phi[\mathrm{rad}]$ & $\pi / 6$ & $\pi / 6$ & $\pi / 6$ \\
\hline
\end{tabular}

Table 4: Parameters for generating functional trajectories focusing on the phase difference. 

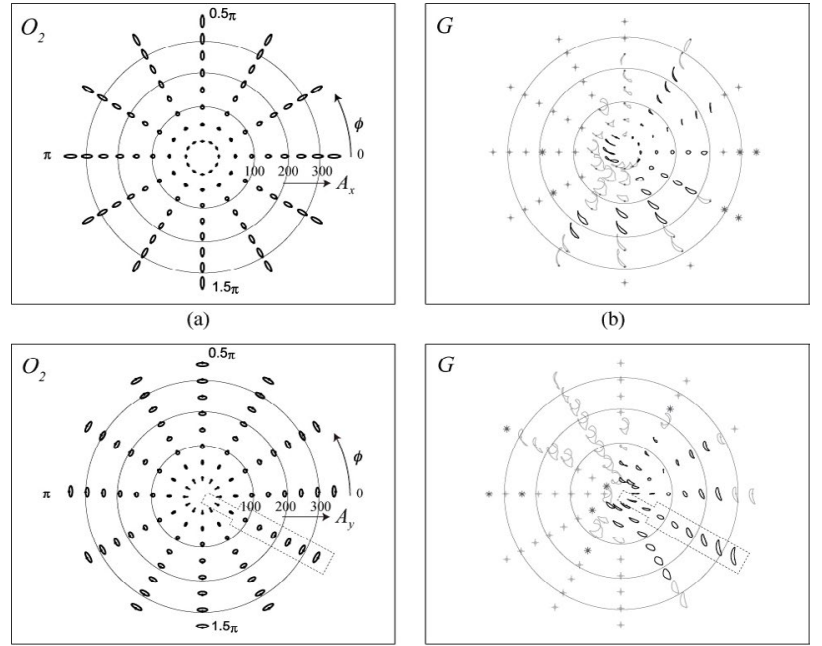

(c)

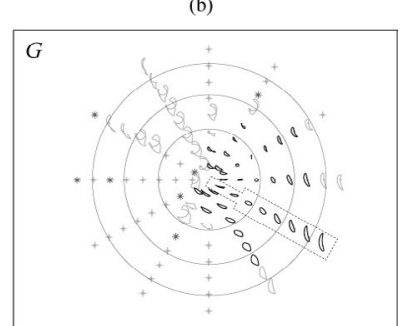

(d)

Figure 9: Dependency of trajectory modifications $\mathbf{G}=[G, G]$ with changes of the $O_{2}$ movement with respect to amplitude parameters $A_{x}$ and $A_{y}$ with eight steps $[0,50,100,150,200,250,300,350] \times 10^{-3} \mathrm{~m}$ and rotation angles $\phi$ rad every $\pi / 6$ rad. Top figures of (a) and (b) represent $A_{x}$ dependency and bottom figures (c) and (d) represent $A y$ dependency with 96 sets ( 8 steps $\times 12$ angles). In the end effector trajectory (b) and (d), black and gray lines represent non-broken and broken trajectories respectively. In comparison of $A_{x}$ and $A_{y}$ dependencies, the total non-broken trajectories of 34 and 35 types respectively were obtained as approximately $35 \%$ consistently, and the fine parameter of $A$ was restricted in a range such as less than $200 \times 10^{-3} \mathrm{~m}$ if $\phi=0$ (b) while a robust $\phi=-\pi / 6 \mathrm{rad}$ was found in $A_{y}$ extension ranging from 0 to $350 \times 10^{-3} \mathrm{~m}$ (bordered by the dotted line). It suggests a limitation of the $A_{x}$ and $A_{y}$ length and a robustness of the $A$ extension with the specific rotation angle $\phi$.

with the horizontal amplitude $A_{y}$ changing from 0 to $350 \times 10^{-3} \mathrm{~m}$ associated with the change of the rotation angle $\phi$ under the fixed condition of the vertical amplitude $A_{x}=100 \times 10^{-3} \mathrm{~m}$. Figure 9 (c) illustrated 96 types of the $\mathrm{O}_{2}$ parametric orbit by the combination of eight amplitudes of $A_{y}$ and twelve rotation angles, which were plotted in concentric circles with respect to those variations, and Figure 9 (d) showed resultant trajectories of the end effector in the same arrangement as well as the previous result. Consistently, generated trajectories included oval, half-moon, and figure-of-eight non-broken shapes. Fine trajectories of 35 types were obtained and the success rate was $36.46 \%$.

In Figure 9 (d), a robust $\phi=-\pi / 6 \mathrm{rad}$ was found in $A_{y}$ extension ranging 0 to $350 \times 10^{-3} \mathrm{~m}$ (bordered by the dotted line). The detailed analysis of the selected parameter range was shown in Figure 10. In this case, a fine elliptic orbit with a sharp edge in front was obtained at $A_{y}=0$, similarly to the walking pattern of the original Theo Jansen mechanism, and it varied in oval shapes which were useful for climbing motions by stretching in the upward direction (climbing-like trajectory analyzed in Figure 5). This result suggests the sensitivity of $\phi$ for providing nonbroken trajectories.

In this section, dependency of trajectory modification was examined with respect to the change of amplitudes $A_{x}$ and $A_{y}$, and the results suggest a limitation of the $A_{x}$ and $A_{y}$ length and a robustness of the $A_{y}$ extension with the specific rotation angle $\phi$.

The limitation of the amplitude is plausible in terms of the ratio with respect to the driving link $O_{1}$ movement. Amplitude sensitivity as a connected area is unclear in the concentric distribution pattern, while

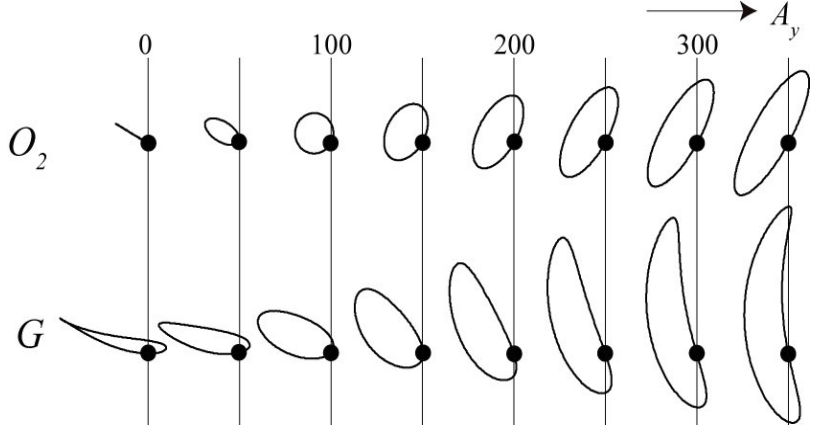

Figure 10: A set of generated trajectories with respect to the change of $\mathrm{O}_{2}$ movements with parameters of the rotation angle $\phi=-\pi / 6 \mathrm{rad}$ in the case of $A_{y}$ enlargement (the bordered area in Figure 9 ). In this case, the elliptic orbit was modified from a shape suitable for walking (close to the original trajectory) to a shape stretching upward (climbing-like trajectory analyzed in Figure 5). This result suggests the sensitivity of $\phi$ for providing non-broken trajectories.

interestingly the rotation angle specificity was found, such as the case $\phi=-\pi / 6 \mathrm{rad}$. Thus, in the next section, the relationship between rotation $\phi$ and phase differences $\delta$ of $O_{2}$ with $O_{1}$ is discussed.

\section{Functional orbit generation focusing on the phase difference}

According to Equation (16) and Equation (17), this section focused on two parameters of the rotation angle of the joint center $\mathrm{O}_{2}$ movement as $\phi$ and the phase difference of the joint center with respect to the $O_{1}$ movement, under the fixed condition of amplitudes $A_{x}$ and $A_{y}$. A simple example of the morphological change of the trajectory with respect to the phase difference $\delta$ (varying from 0 to $11 \pi / 6 \mathrm{rad}$ ) was shown in Figure 11, which was obtained using parameters in the first column of Table 4.

In the case of the $\phi-\delta$ variation, various trajectories were examined with the phase difference of every $\pi / 6$ rad step associated with the change of the rotation angle $\phi$ under the fixed condition of the vertical amplitude $\left[A_{x}, A_{y}\right]=[50,100] \times 10^{-3} \mathrm{~m}$ using parameters in the second column of Table 4 as a set of ellipses stretched in the tangential direction against the concentric circle. Figure 12 (a) illustrated 144 types of the $\mathrm{O}_{2}$ parametric orbit by the combination of twelve steps of and twelve rotation angles $\phi$, which were plotted in concentric circles with respect to those variations, and Figure 12 (b) showed resultant trajectories of the end effector in the same arrangement. Similar types of generated trajectories were obtained as well as the previous analyses. Fine trajectories of 70 types were obtained and the success rate was $41.67 \%$. According to the change of $\phi$, generated trajectories were obtained as regular patterns. Non-broken trajectories were obtained if $\phi$ is ranging from $-2 \pi / 6$ to $2 \pi / 6 \mathrm{rad}$. On the other hands, broken trajectories were generated when angle ranges as $\pi / 2 \leq \phi \leq 3 \pi / 2 \mathrm{rad}$.

In the case of the $\phi-\delta$ variation under the fixed condition of the vertical amplitude $\left[A_{x}, A_{y}\right]=[100,50] \times 10^{-3} \mathrm{~m}$, parameters in the last column of Table 4 were used as ellipse stretched in the normal direction against the concentric circle. Figure 12 (c) illustrated 144 types of the $\mathrm{O}_{2}$ parametric orbit by the combination of twelve steps of and twelve $\delta$ rotation angles $\phi$, which were plotted in concentric circles with respect to those variations, and Figure 12 (d) showed resultant trajectories of the end effector in the same arrangement. Similar types of generated trajectories were obtained as well as the previous analyses. Fine trajectories of 71 types were obtained and the success rate was $54.17 \%$. 
1

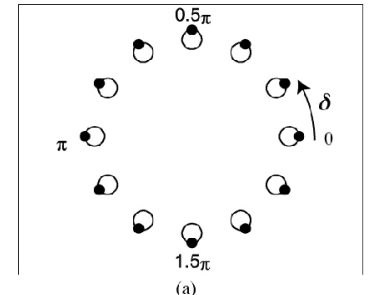

(a)

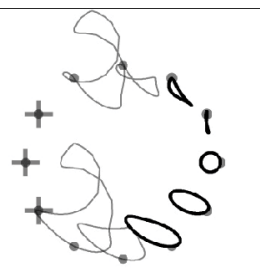

(b)
Figure 11: Definition of the phase difference of the $\mathrm{O}_{2}$ movement with respect to the driving link $O_{1}$, abbreviated as $\delta$ (a). Even in the same condition with amplitudes $A_{\mathrm{x}}=A_{\mathrm{y}}=100 \times 10^{-3} \mathrm{~m}$ and rotation angle $\phi=0$, a sensitivity with the phase difference $\delta$ influences the generation of nonbroken trajectories as shown in (b).

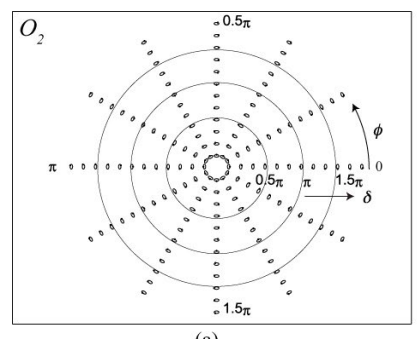

(a)

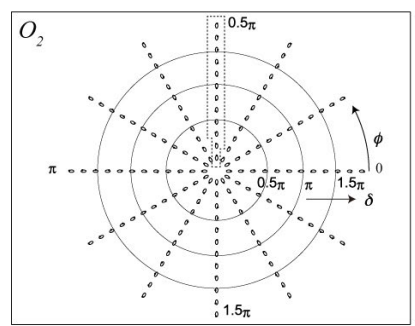

(c)
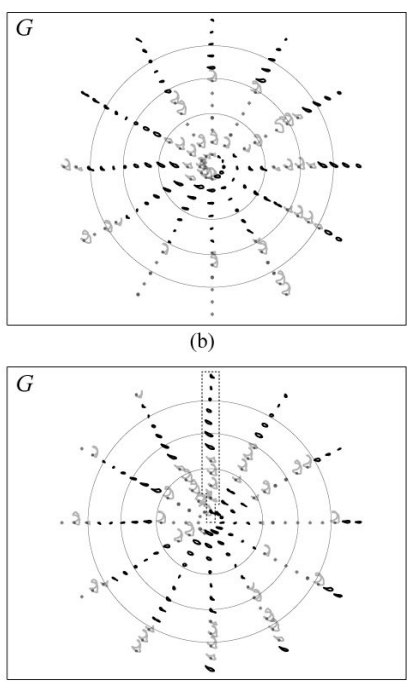

(d)
Figure 12: Dependency of trajectory modifications $\mathbf{G}=\left[G_{x^{\prime}}, G_{y}\right]$ with changes of the $\mathrm{O}_{2}$ movement with respect to amplitude parameters $\phi$ and $\delta$. Rotation angles $\phi$ was given every $\pi / 6$ and $\delta$ was given as twelve steps every $\pi / 6$ rad. Amplitude parameters were given as $\left[A_{x}, A_{y}\right]=[50,100]$ in top figures of (a) and (b), and $\left[A_{x^{\prime}} A_{y}\right]=[100,50]$ in bottom figures of (c) and (d) with 144 sets (12 steps $\times 12$ angles). In the end effector trajectory (b) and (d), black and gray lines represent non-broken and broken trajectories respectively. In comparison of and $A$ dependencies, the total non-broken trajectories of 70 and 71 types respectively were obtained as approximately $50 \%$ consistently, and the fine parameter exists in some range of $\phi$.

According to changing $\phi$, generated trajectories were obtained as regular patterns. According to the change of $\delta$ and $\phi$, generated trajectories varied as cyclic patterns. Non-broken trajectories were obtained, when $\delta-\phi$ is from $\pi / 6$ to $7 \pi / 6 \mathrm{rad}$.

In Figure $12(\mathrm{~d})$, trajectories with $\phi=\pi / 2 \mathrm{rad}$ were examined as a sample to analyze the sensitivity with $\delta$ (bordered by the dotted line) and the detail analysis was shown in Figure 12. The detail analysis of the selected parameter range was shown in Figure 13. In this case, broken trajectories were found in the range $[\pi / 6,4 / 6 \pi] \mathrm{rad}$ while non-broken trajectories were obtained in others, which represented the $\delta$ sensitivity. In the comparison with the amplitude modification, which had a scattered distribution of non-broken trajectories and thus is unable to specify a consistent area, this result indicates that the modification using the parameter $\delta$ can be used for systematic change of the nonbroken trajectories, avoiding singular configurations.

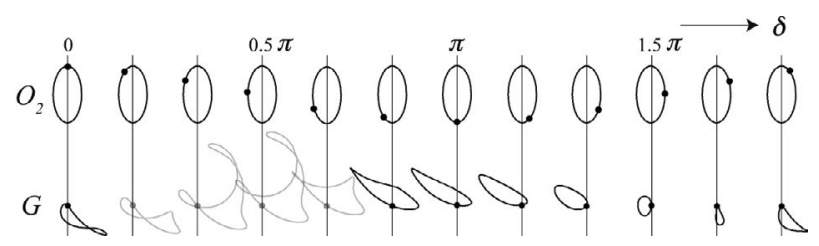

Figure 13: A set of generated trajectories with respect to the change of $\mathrm{O}_{2}$ movements with parameters of the rotation angle $\phi=\pi / 2$ rad in the case of $\delta$ change (the bordered area in Figure 12). In this case, broken trajectories were found in the range $[\pi / 6 ; 4 / 6 \pi]$ rad while non-broken trajectories were obtained in others, related to the $\delta$ sensitivity.

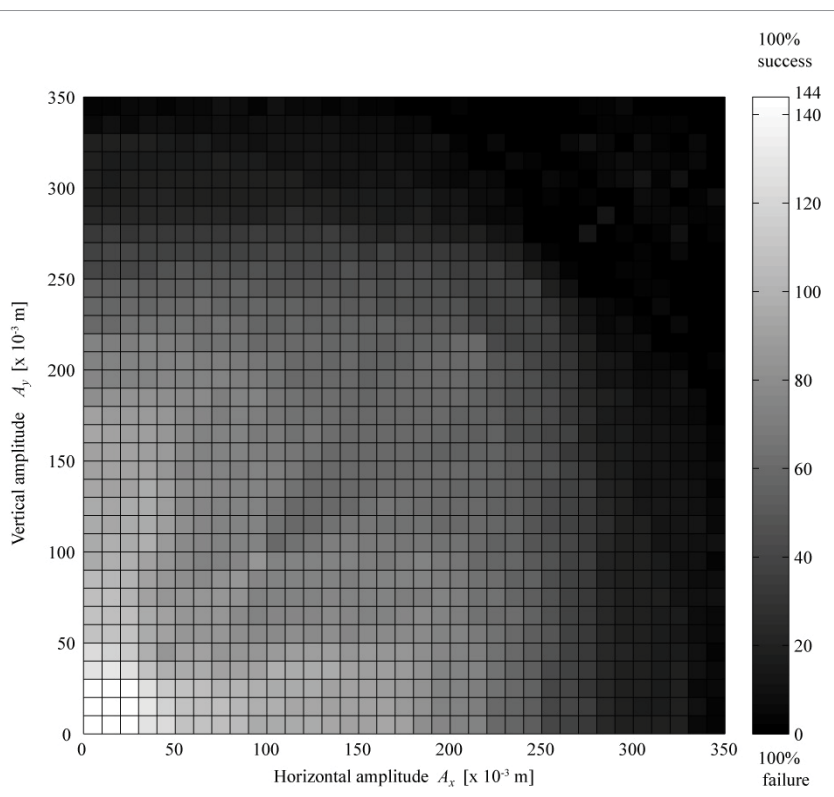

Figure 14: Contour map of $A_{x}-A_{y}$ dependencies of non-broken trajectories, which were given by individual success rates with respect to the change of the horizontal amplitude $A_{x}$ and vertical amplitude $A_{y}$.

\section{Systematic analyses in four controllable parameters}

As the final analyses, we focus on the discussion of the success rate to generate non-broken trajectories from the extended Theo Jansen mechanism in the viewpoint of four parameters with amplitudes $A_{x}$ and $A_{y}$, rotation angle of the $O_{2}$ movement $\phi$, and the phase difference $\delta$ between $O_{2}$ and $O_{1}$ movements. In this section, non-broken trajectories were analyzed by changing $A_{x^{\prime}} A_{y}, \delta$ and $\phi$ respectively according to Table 5.

With respect to parameters of amplitudes $A_{x}$ and $A_{y}$ varying from 0 to $350 \times 10^{-3} \mathrm{~m}$, total 144 types conditions were examined whether they were broken or not to calculate the success rate of the condition. Figure 14 showed the success rate of every condition. White pixels represent the complete success condition without any broken trajectories and black pixel represents complete failure with broken trajectories only. Edges defined by $75 \%$ success rate was found around amplitudes $A_{x}$ and $A_{y}$ equal to $40 \times 10^{-3} \mathrm{~m}, 58 \%$ success rate was found around $A_{x}=A_{y}=100$ $\times 10^{-3} \mathrm{~m}, 42 \%$ success rate was found around $A_{x}=A_{y}=110 \times 10^{-3} \mathrm{~m}, 25 \%$ success rate was found around $A_{x}=A_{y}=230 \times 10^{-3} \mathrm{~m}$, and $0 \%$ success rate was found around amplitudes $A_{x}$ and $A_{y}$ equal to $260 \times 10^{-3} \mathrm{~m}$.

With respect to parameters of the rotation angle $\phi$ and the phase 


\begin{tabular}{|c|c|}
\hline Parameter & Value \\
\hline$A_{x}\left[\times 10^{-3} \mathrm{~m}\right]$ & $0 \leq A_{x} \leq 350$ \\
\hline$d A_{x}\left[\times 10^{-3} \mathrm{~m}\right]$ & 10 \\
\hline$A_{y}\left[\times 10^{-3} \mathrm{~m}\right]$ & $0 \leq A_{y} \leq 350$ \\
\hline$d A_{y}\left[\times 10^{-3} \mathrm{~m}\right]$ & 10 \\
\hline$\omega[\mathrm{rad} / \mathrm{s}]$ & $\pi / 2$ \\
\hline$t[\mathrm{~s}]$ & $0 \leq t \leq 4$ \\
\hline$d t[\mathrm{~s}]$ & $1 / 90$ \\
\hline$\delta[\mathrm{rad}]$ & $0 \leq \delta \leq 11 \pi / 6$ \\
\hline$d \delta[\mathrm{rad}]$ & $\pi / 6$ \\
\hline$\phi[\mathrm{rad}]$ & $0 \leq \phi \leq 11 \pi / 6$ \\
\hline$d \phi[\mathrm{rad}]$ & $\pi / 6$ \\
\hline
\end{tabular}

Table 5: Parameters on distribution of non-broken trajectories.

difference varying from 0 to $11 \pi / 6$ rad every $\pi / 6 \mathrm{rad}$, total 906 types' conditions were examined whether or not broken to calculate the success rate of the condition. Figure 15 showed the success rate of every condition. White pixels represent the complete success condition without any broken trajectories and black pixel represents complete failure with broken trajectories only. The whitest pixel represents the success rate with $74 \%$.

In this systematic analyses of how the extended Theo Jansen mechanism generates non-broken trajectories avoiding the singularity configuration, the parametric oval of the joint center $\mathrm{O}_{2}$ was validated and, as the new finding, necessary conditions not only rely on the limitation of the amplitudes of the oval size but more significantly rely on the linear relationship between the rotation angle $\phi$ and phase difference as shown in Figure 15 exhibiting an inverse proportion.

\section{Discussion}

\section{Similarity and dissimilarity with the biological motion}

In the present analysis, the extensibility of the Theo Jansen mechanism was analyzed using multibody dynamics $[19,20]$ and the reproduction of motions that extensionally-defined from the original walking pattern was demonstrated by releasing the lock of the joint center $\mathrm{O}_{2}$ and introducing the parametric oval.

As is discussed in Section of "Results of Numerical Simulations," insect motions have been widely analyzed [32,33], and applied to robotics known as bio-inspired robotics [38-40,42]. In the analysis of the human motion trajectory, Ivanenko [43] investigated various gait trajectories and decomposed them into principal components, which enabled to reproduce the original trajectories, such as walking, running, hopping, crouched walking, obstacle avoidance, air-stepping and figureof-eight motions in running. Theo Jansen mechanism was highlighted in the similarity of the biological motion, which is still unclear what kinds of characteristics are similar [24]. In the viewpoint of similarity to the trajectory shape, the extended Theo Jansen mechanism can provide a figure-of-eight trajectory in gait, while the linkage mechanism is designed by linkages based on the mechanical engineering which is not simply corresponds to the human musculoskeletal system. It is quite different in the viewpoint of the physical property. Interestingly, the present result revealed that a specific relationship between the $\mathrm{O}_{2}$ and $O_{1}$ as the phase difference $\delta$ and rotation angle $\phi$ provided a simple set of variables to enable the system to be controlled for generating various functional motions even if it was the complex system. Assumptions of the biological mechanism to control body parts in the phase-rotationamplitude parameter space was frequently discussed in the model using phase-coupling oscillators having synchronization and body coordination
[15]. Such a control mechanism has the capability to generate an extended behavior by adding a new component of the synchronous oscillator [18]. The present result demonstrates the importance of the coupling property coordinating between the body and the controller (nervous system) through the phase and rotation even in a physically different linkage system.

\section{Singularity analysis in the linkage system}

Singularity analyses have been investigated in the parallel linkage system especially for planer linkages and manipulators [4450]. A geometrically and physically based method for the detection of singularities was proposed [48], which provided information representing the distance of a given position to singularities. In the planer robot with multi legs, the singularity detection associated with a robot controller is useful for a path planning algorithm avoiding singularities during the locomotion. In order to find an appropriate path, the workspace can be defined as a virtual potential field $[48,49]$ and such a method can be applied to the real-time control.

In a planar parallel manipulator, Gosselin and Angeles [44] established the mathematical foundations with Jacobian matrices of the chain and demonstrated the applicability to planar RRRP mechanism, the Watt linkage, planar three-degree-of-freedom manipulator with revolute actuators and spherical three-degree-of-freedom parallel manipulator. The extended Theo Jansen mechanism can be considered as an extension of the Watt linkage and our method of the singularity detection has a further theoretical perspective with respect to their method. Focusing on the geometrical property, Liu [47] proposed a method for detection of the singularity in parallel manipulators, by using differential topology strongly influenced by the Morse theory [51]. Morse theory analyzes the topology of a manifold by focusing on differentiable functions on the target manifold, and it allows the target to find a specific topological structure and handle decompositions on manifolds based on their homology, like a part of the torus as a simple example. In the present study, we found the phase-rotation relationship in the parameter space to detect the singularity, which can be considerable in the topological feature extracting from descriptions

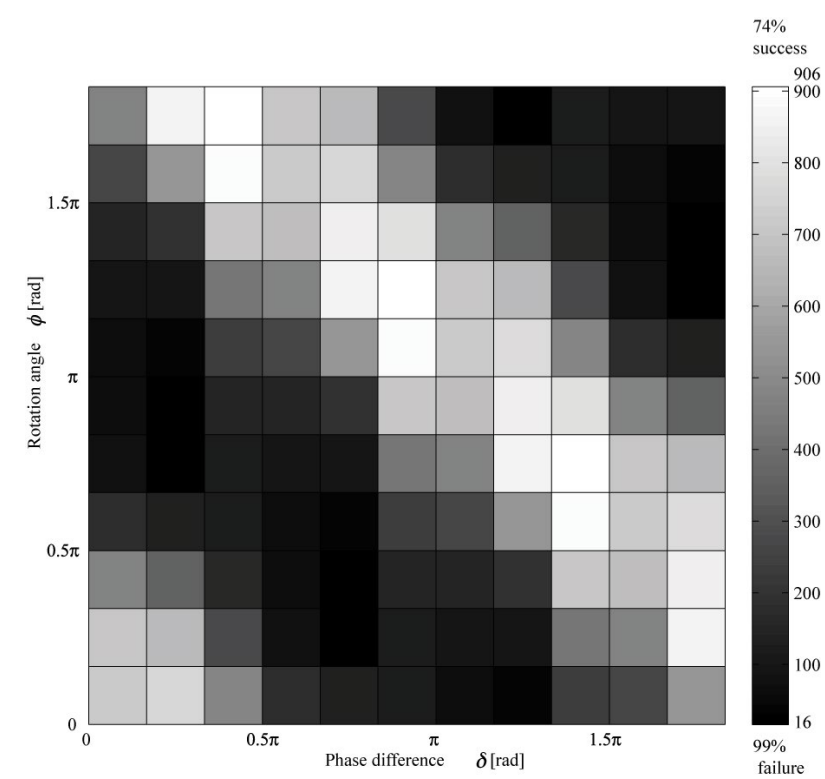

Figure 15: Contour map of $\delta-\phi$ dependencies of non-broken trajectories, which were given by individual success rates with respect to the change of the phase difference $\delta$ and rotation angle $\phi$. 
Citation: Komoda K, Wagatsuma H (2015) A Determinant Analysis to Detect the Singularity of the Extended Theo Jansen Mechanism in the PhaseRotation-Amplitude Parameter Space. J Comput Sci Syst Biol 9:1 010-022. doi:10.4172/jcsb.1000215

using generalized coordinates based on the physical context of the kinematics and dynamics.

\section{Conclusion}

In the present study, multibody dynamics was introduced to investigate the singularity of the extended Theo Jansen mechanism with respect to the parametric oval given as a new freedom point at the joint center $\mathrm{O}_{2}$. The method realized the system to exhibit the extendibility in terms of flexibility in the gait trajectory, including climbing-like, figureof-eight and dropping-down motions. In the systematic numerical simulation, the extended Theo Jansen mechanism reproduced various new functional gait trajectories, associated with four parameters with amplitudes of the parametric oval, its rotation angle and the phase difference between the joint center $\mathrm{O}_{2}$ and the driving link $O_{1}$ movements, coupling with the proposed determinant analysis to detect the singularity and dead lock of the system. Importantly, a specific inverse proportional relationship between the rotation angle $\phi$ and phase difference $\delta$ in the contour map of $\delta-\phi$ dependencies of nonbroken trajectories is established, which csts of individual success rates under every condition. To prevent the singularity of the extended Theo Jansen mechanism, necessary conditions not only rely on the limitation of the amplitudes of the oval size but more rely significantly on the phase-rotation relationship.

\section{Acknowledgements}

This work was partly supported by Council for Science, Technology and Innovation, "Cross-ministerial Strategic Innovation Promotion Program (SIP) Infrastructure Maintenance, Renovation, and Management". (funding agency: NEDO)

\section{References}

1. Sitti M, Menciassi A, ljspeert AJ, Low KH, Kim S (2013) Survey and introduction to the focused section on bio-inspired mechatronics. Mechatronics IEEE/ASME Transactions 18: 409-418.

2. McGeer T (1990) Passive dynamic walking. The international journal of robotics research 9: 62-82.

3. Schwab AL, Wisse M (2001) Basin of attraction of the simplest walking model. Proceedings of the ASME Design Engineering Technical Conference 6: 531539.

4. Blajer W, Krzysztof D, Zenon M (2007) Multibody modeling of human body for the inverse dynamics analysis of sagittal plane movements. Multibody System Dynamics 18: 217-232.

5. Funabashi H, Ogawa K, Gotoh Y, Kojima F (1984) Synthesis of leg mechanisms of biped walking machine (Part I, Synthesis of Ankle-PathGenerator), Transactions of the Japan Society of Mechanical Engineers Series C 50: 1292-1297.

6. Shieh WB, Tsai LW, Azarm S (1997) Design and optimization of a one-degreeof-freedom six-bar leg mechanism for a walking machine. Journal of Robotic systems 14: 871-880.

7. T. Jansen (2007) Theo Jansen: The Great Pretender. Nai Publishers, Rotterdam.

8. Giesbrecht D, Wu CQ, Sepehri N (2012) Design and Optimization of an EightBar Legged Walking Mechanism Imitating a Kinetic Sculpture, "Wind Beast". Transactions of the Canadian Society for Mechanical Engineering 36: 343-355.

9. Kim SW, Jung H, Kim Y, Kim H, Han SH, et al. (2010) Analysis and Design of a Legged Walking Robot Based on Jansen Mechanismm. SCIS \& ISIS 2010: 920-924.

10. Sitharam M, Wang M (2014) How the Beast really moves: Cayley analysis of mechanism realization spaces using CayMos. Computer-Aided Design 46: 205-210

11. Nansai S, Rojas N, Elara MR, Sosa R, Iwase M (2015) On a Jansen leg with multiple gait patterns for reconfigurable walking platforms. Advances in Mechanical Engineering 7: 1-18.
12. Komoda K, Wagatsuma $H$ (2012) A proposal of the extended mechanism for Theo Jansen linkage to modify the walking elliptic trajectory and a study of cyclic base function. Dynamic Walking Conference, Ohio.

13. Komoda K, Wagatsuma $H$ (2014) Singular configurations analyses of the modifiable Theo Jansen-like mechanism by focusing on the Jacobian determinant - A finding limitations to exceed normal joint range of motion. In Advanced Intelligent Mechatronics (AIM), 2014 IEEE/ASME International Conference, Besacon.

14. ljspeert AJ (2008) Central pattern generators for locomotion control in animals and robots: a review. Neural Netw 21: 642-653.

15. Taga G, Yamaguchi Y, Shimizu H (1991) Self-organized control of bipedal locomotion by neural oscillators in unpredictable environment. Biological Cybernetics 65: 147-159.

16. Cappellini G, Ivanenko YP, Poppele RE, Lacquaniti F (2006) Motor patterns in human walking and running. J Neurophysiol 95: 3426-3437.

17. Rybak IA, Shevtsova NA Lafreniere-Roula M, McCrea DA (2006) Modelling spinal circuitry involved in locomotor pattern generation: in-sights from deletions during fictive locomotion. The Journal of physiology 577: 617-639.

18. Aoi S, Kondo T, Hayashi N, Yanagihara D, Aoki S, et al. Contributions of phase resetting and interlimb coordination to the adaptive control of hindlimb obstacle avoidance during locomotion in rats: a simulation study. Biological cybernetics 107: 201-216, 2013

19. Nikravesh PE (1988) Computer-aided analysis of mechanical systems. Englewood Cliffs, New Jersey.

20. Haug EJ (1989) Computer Aided Kinematics and Dynamics of Mechanical Systems/Vol.1 Basic Methods. Ally and Bacon, Boston.

21. Schiehlen W (1990) Multibody systems handbook. Springer, Berlin.

22. Shabana AA (1989) Dynamics of multibody systems. Cambridge university press, New York.

23. Yoshikawa T (1990) Foundations of robotics: analysis and control. The MIT Press, London.

24. Ghassaei A (2011) The Design and Optimization of a Crank-Based Leg Mechanism. Senior Thesis, Pomona College, USA.

25. Seeni A, Schafer B, Hirzinger G (2010) Robot mobility systems for planetary surface exploration-state-of-the-art and future outlook: A literature survey. In Aerospace Technologies Advancements, INTECH Open Access Publisher.

26. Klaassen B, Linnemann R, Spenneberg D, Kirchner F (2002) Biomimetic walking robot SCORPION: Control and modeling. Robotics and autonomous systems 41: 69-76.

27. Gorner M (2007) Mechatronic Concept of Crawler from DLR-Hand II Technology. Diploma Thesis, Institute of Robotics and Mechatronics, German Aerospace Center, Germany.

28. Apostolopoulos DS (2001) Analytic Configuration of Wheeled Robotic Locomotion. PhD Thesis, Carnegie Mellon University, USA.

29. Besseron G, Grand C, Amar FB, Plumet F, Bidaud P (2005) Loco-motion modes of an hybrid wheel-legged robot. Climbing and Walking Robots, Springer Berlin, Heidelberg

30. Rehmat Z, Roll J, Lee JS, Yim W, Trabia MB (2009) Design of "Figure-8" Spherical Motion Flapping Wing for Miniature UAV. ASME 2009 International Design Engineering Technical Conferences and Computers and Information in Engineering Conference, Las Vegas, USA.

31. Masoomi SF, Gutschmidt S, Chen X (2014) State of the Art in Biomimetic Swimming Robots: Design Principles and Case Study. Handbook of Research on Advancements in Robotics and Mechatronics, Pennsylvania, USA.

32. Sane SP (2003) The aerodynamics of insect flight. J Exp Biol 206: 4191-4208.

33. Dickinson MH, Lehmann FO, Sane SP (1999) Wing rotation and the aerodynamic basis of insect flight. Science 284: 1954-1960.

34. Ellington CP (1999) The novel aerodynamics of insect flight: applications to micro-air vehicles. J Exp Biol 202: 3439-3448.

35. Keller JB, Rubinow SI (1976) Swimming of flagellated microorganisms. Biophys J 16: 151-170.

36. Pikovsky A, Rosenblum M, Kurths J (2013) Synchronization: a universa concept in nonlinear sciences. Cambridge University press, New York, USA. 
Citation: Komoda K, Wagatsuma H (2015) A Determinant Analysis to Detect the Singularity of the Extended Theo Jansen Mechanism in the PhaseRotation-Amplitude Parameter Space. J Comput Sci Syst Biol 9:1 010-022. doi:10.4172/jcsb.1000215

37. Zbikowski R, Galinski C, Pedersen CB (2005) Four-bar linkage mechanism for insectlike flapping wings in hover: Concept and an outline of its realization. Journal of mechanical design 127: 817-824.

38. Yang LJ (2012) The micro-air-vehicle Golden Snitch and its figure-of-8 flapping. Journal of Applied Science and Engineering 15: 197-212.

39. Wen L, Lauder G (2013) Understanding undulatory locomotion in fishes using an inertia-compensated flapping foil robotic device. Bioinspiration \& biomimetics 8: 1-15.

40. Was L, Lauga E (2014) Optimal propulsive flapping in Stokes flows. Bioinspir Biomim 9: 016001.

41. Galinski C, Zbikowski R (2005) Insect-like flapping wing mechanism based on a double spherical Scotch yoke. J R Soc Interface 2: 223-235.

42. Tadano S, Takeda R, Miyagawa $H$ (2013) Three dimensional gait analysis using wearable acceleration and gyro sensors based on quaternion calculations. Sensors 13: 9321-9343.

43. Ivanenko YP, Cappellini G, Dominici N, Poppele RE, Lacquaniti F (2007) Modular control of limb movements during human locomotion. J Neurosci 27 : $11149-11161$.
44. Gosselin C, Angeles J (1990) Singularity analysis of closed-loop kine-matic chains. Robotics and Automation, IEEE Transactions on 6: 281-290.

45. St-Onge BM, Gosselin CM (2000) Singularity analysis and representation of the general Gough-Stewart platform. The International Journal of Robotics Research 19: 271-288.

46. Kong K, Gosselin CM (2002) Kinematics and singularity analysis of a novel type of 3-CRR 3-DOF translational parallel manipulator. The International Journal of Robotics Research 9: 791-798.

47. Liu G, Lou Y, Li Z (2003) Singularities of parallel manipulators: a geometric treatment. Robotics and Automation, IEEE Transactions on 19: 579-594.

48. Dietrich F, Maa J, Bier C, Pietsch I, Raatz A, et al. (2011) Detection and avoidance of singularities in parallel kinematic machines. In Robotic Systems for Handling and Assembly, Springer, Berlin, Heidelberg.

49. Schutz D, Wahl FM (2010) Robotic Systems for Handling and As-sembly. Springer Tracts in Advanced Robotics, Springer-Verlag, Berlin, Heidelberg.

50. Zhao J, Feng Z, Ma N, Chu F (2014) Workspace and Singularity of Planar Parallel Linkage. In Design of Special Planar Linkages, Springer, Berlin, Heidelberg.

51. Milnor JW (1963) Morse Theory. Princeton university press, New Jersey, USA. 